\title{
Participación ciudadana y cohesión social: intervención comunitaria en asentamiento humano de la ciudad de Durango México
}

\author{
Iván Rafael Vázquez Serrano \\ rafaelvs@ujed.mx \\ https://orcid.org/0000-0002-7795-9506 \\ Universidad Autónoma de Nayarit \\ José Gerardo Ignacio Gómez Romero \\ igomez@ujed.mx \\ https://orcid.org/0000-0002-6322-6133 \\ Universidad Juárez del Estado de Durango \\ César Alberto Gurrola Pérez \\ cesaralberto.gurrola@ujed.mx \\ https://orcid.org/0000-0002-7739-5341 \\ Universidad Juárez del Estado de Durango
}

\section{RESUMEN}

En el presente documento se reportan los resultados de una intervención comunitaria realizada con el fin de generar una participación ciudadana activa en una comunidad y analizar la relación con su cohesión social. Esta intervención se llevó a cabo en la Colonia Legisladores de la ciudad de Durango, México, asentamiento humano que mostraba síntomas de descomposición social y abandono de las autoridades. Se aplicó mediante la metodología de la investigación acción participativa, apoyándose en las etapas: diagnóstico, diseño, implementación y evaluación. Destacando que la intervención comunitaria logró aumentar la participación ciudadana de un nivel de información a uno de colaboración y de la cohesión social, de un nivel de lazos sociales a uno de confianza. Se concluye que en la medida en que los ciudadanos se hagan partícipes de manera comunitaria del mejoramiento de las condiciones de su entorno, aumentarán sus lazos, su confianza, sus valores y su sentido de pertenencia, concibiendo la cohesión social como un constructo que se da desde la interacción social y no desde la individualidad.

Palabras clave: cohesión social; participación ciudadana; intervención; comunidad. 


\title{
Citizen participation and social cohesion: community intervention in human settlement in the city of Durango, Mexico
}

\begin{abstract}
This document reports the results of the community intervention carried out in order to generate citizen participation in a community and analyze the relationship with its social cohesion. This intervention was carried out in the Legisladores neighborhood of the city of Durango, México, A human settlement that showed symptoms of social decomposition and abandonment of the authorities. It was applied through the participatory action research methodology, based on the stages: diagnosis, design, implementation and evaluation. Emphasizing that community intervention managed to increase citizen participation from a level of information to one of collaboration and of social cohesion from a level of social ties to one of trust. It is concluded that to the extent that citizens participate in a community way in the improvement of the conditions of their environment, their ties, their trust, their values and their sense of belonging will increase, as dimensions of social cohesion, conceiving social cohesion as a construct that occurs from society and not from individuality.
\end{abstract}

Keywords: social cohesion; citizen participation; intervention; community.

Artículo recibido: 30 noviembre. 2021 Aceptado para publicación: 29 diciembre 2021

Correspondencia: rafaelvs@ujed.mx Conflictos de Interés: Ninguna que declarar 


\section{INTRODUCCIÓN}

A finales del siglo XX e inicios del XXI, comenzaron a darse profundos cambios en el entorno, provocados por el fenómeno de la "globalización", propiciando lo que llama Gómez (2016) como un "cambio de época", debido a un cúmulo de transformaciones repentinos que se dieron en diversos aspectos de la dinámica mundial, propiciados entre varios factores, por la detonación tecnológica de la cuarta revolución industrial (Oliván, 2014), que vino a alterar nuestro modo de comunicarnos y de convivir.

La sociedad está en una nueva era que ha traído consigo una transformación en el modo de ver, conceptualizar y materializar la vida en comunidad, y ha generado nuevos paradigmas de socialización y asociación del ser humano, impactando radicalmente en la dinámica organizacional de nuestro mundo, en donde la posmodernidad y las nuevas tecnologías de la información y la comunicación, han provocado un peculiar fenómeno de acercar a los que están lejos, pero alejar a los que están cerca, profundizando lo que venimos presenciando las últimas décadas, y que Paz y Miño (2017), llama la “deshumanización de la sociedad".

La colonia Legisladores de la ciudad de Durango, México, es un asentamiento humano que se desarrolla en un ambiente de descomposición social, en donde domina la indiferencia, el conflicto, la violencia desde el hogar hasta las calles, así como la inseguridad que provocan los grupos pandilleriles; sin dejar de lado las condiciones generalizadas de pobreza y marginación. La zona de la ciudad donde se ubica esta colonia está catalogada por las autoridades locales como una de las más peligrosas por su alta incidencia delictiva (Lastra, 2017).

Por este motivo, se decidió llevar a cabo una intervención comunitaria en esa colonia, que incentivara la participación ciudadana y generara un cambio de actitud de la comunidad, que incrementara su cohesión social. De manera tal que se planteó un objetivo de investigación que busca medir la influencia de la participación ciudadana en la cohesión social de los habitantes de la colonia legisladores de la ciudad de Durango, México; y que de respuesta a la pregunta ¿de qué manera influye la participación ciudadana en la cohesión social de la comunidad estudiada? y a la hipótesis: el aumento de la participación ciudadana de la población, en los asuntos de su propio entorno, genera un incremento en la cohesión social de la Colonia Legisladores de la ciudad de Durango, México. 


\section{REVISIÓN DE LITERATURA}

Las organizaciones tienen una naturaleza eminentemente social y están presentes completamente en la vida del ser humano, como afirma Hall (1996) nacemos, crecemos, vivimos y nos reproducimos en organizaciones, como lo son los hospitales, las escuelas, el gobierno, las empresas, y son inevitables e indiscutiblemente necesarias para el desarrollo y complementariedad de las personas que conviven y se desarrollan en estas. Conforme ha ido evolucionando el hombre, ha transformado la manera de organizarse en sociedad, desde la horda, la tribu, el clan, hasta llegar a lo que ahora conocemos como la ciudad, y dentro de ellas las "comunas" que en nuestra cultura las conocemos como los "asentamientos humanos", que, en este sentido, son un tipo de organización.

Después de la crisis de gobernabilidad del Estado como lo afirma Aguilar (2007), de la incapacidad del Estado para gestionar las crisis (Vargas, 2007) y la fragmentación de la sociedad como lo señala Guerrero (2008), estas organizaciones al sentirse marginadas y excluidas, toman posturas distintas, algunas se aíslan y no se organizan, pero otras si lo hacen, buscando resolver sus propias necesidades y alcanzar con una serie intereses en común.

De esta manera, surge la acción de organizarse en comunidad, es decir, un grupo de personas que agrupadas en una comuna, colonia o ciudad, a los que llamamos ciudadanos, que buscan la conformación de espacios públicos como puntos de encuentro para transformar la realidad de su entorno (Borja, 2000), ciudadanos activos que se apropian de sus derechos y los ejercen, a la vez que asumen sus responsabilidades ante su comunidad (Olvera, 2019), manifestándose lo que se conoce como participación ciudadana.

\subsection{Participación Ciudadana}

La participación ciudadana representa un acto social, de pertenecer a una entidad organizacional, conformada por dos o más personas, pues no se puede participar de manera individual, para sí mismo. De esta manera la participación se encuentra en el centro de la sociedad, como una fuerza moral que impulsa a los individuos a buscar la colaboración con otros para mejorar sus propias circunstancias.

La participación ciudadana se entiende pues como la acción colectiva de los actores sociales e institucionales, de integrarse, proponer, controlar y evaluar críticamente los procesos de decisión pública en las esferas territoriales (Lechner, 1991; Zimmerman, 
1992). En este sentido, la participación toma un sentido social, en donde la ciudadanía para hacerse efectiva pasa de una naturaleza individual a una colectiva. (Zimmerman, 1992)

Nuria Cunill (1991, p. 49) define la participación ciudadana como "la intervención de la sociedad civil en la esfera de lo público”. De manera similar, Alberich y Espadas (2014, p. 18), afirma que "es el conjunto de estructuras organizativas, actividades, procesos y técnicas por los que la población interviene en los asuntos públicos que le afectan”.

De esta manera, la participación ciudadana, aboca a la asociación colectiva, desde el conjunto de ciudadanos que conforman una comunidad y se preocupan por el desarrollo de su espacio público para el interés general. Este fenómeno puede influir en el aumento de cohesión social de su entorno.

\subsection{Cohesión Social}

Por otro lado, la cohesión social es el elemento indispensable que mantiene las relaciones y la integración de toda sociedad (Durkheim 1987, en Jiménez, 2013). Cohesión, etimológicamente proviene de, "cohaesus" que significa estar juntos, unidos, adheridos a algo, unión de partes, que en español significa "unión y relación adecuada de todas las partes que forman un todo"; y al agregarle el adjetivo social, sería: "unión y relación armoniosa de todas las partes de una sociedad", refiriéndose a los actos voluntarios para asegurar la vida en común, es decir, garantizar el proceso civilizador de una comunidad (Cattani, 2011).

Vera (2014) y Nari (2015), señalan que de acuerdo a las contribuciones académicas y político-institucionales que se han hecho sobre la cohesión social, se puede hacer una clasificación respecto al enfoque en que estas se han postulado, apreciándose la conformación de estos tres enfoques principales:

El enfoque normativo, expone una cohesión social como un modelo deseable, en el cual existe un acuerdo político-institucional, para crear un sistema que genere políticas públicas que busquen garantizar la igualdad, el bienestar y la inclusión social entre los ciudadanos. En este enfoque entran principalmente los postulados de instituciones internacionales como gubernamentales.

El enfoque histórico, el cual defiende la idea de identificar los factores particulares que mantienen unida a cada sociedad, adoptando una perspectiva sociohistórica de la cohesión social, en donde más allá de las recetas y los manuales, cada comunidad tiene sus propias 
características y carencias, por lo que la manera de buscarla será variante. En este enfoque entran los esfuerzos que se han hecho en Latinoamérica para el estudio y la búsqueda de la cohesión.

El enfoque sociológico, en el que se dan las formulaciones propias del aspecto social, sin meterse en otros como el económico. Entran los vínculos sociales, la confianza, los valores compartidos y el sentido de pertenencia. En este grupo, se consideran los estudios cercanos a la propuesta de Émile Durkheim, creador del concepto de cohesión social.

Como lo refleja la tabla 1. se puede ver la diferencia de los diferentes enfoques que se han dado en el estudio y la aplicación de la cohesión social desde diferentes visiones, de las que se derivan los problemas a tratar y las dimensiones con las que se puede solución. En el enfoque normativo podemos observar que los principales problemas y dimensiones tienen una naturaleza inclinada a lo económico, en caso del enfoque histórico, se puede notar una orientación a los motivos contextuales que llevan al conflicto y a la desintegración social; y por último en el enfoque sociológico, podemos ver un sentido meramente social.

Tabla 1. Enfoques de la cohesión social.

\begin{tabular}{|c|c|c|c|}
\hline Enfoque & Exponentes & Problemas & Dimensiones \\
\hline Normativo & $\begin{array}{c}\text { UE, OCDE, } \\
\text { CONEVAL, } \\
\text { Barba. }\end{array}$ & $\begin{array}{c}\text { Exclusión, desigualdad, } \\
\text { polarización, } \\
\text { desconfianza. }\end{array}$ & $\begin{array}{c}\text { Capital social, } \\
\text { exclusión/Inclusión, } \\
\text { brechas económicas, } \\
\text { confianza. }\end{array}$ \\
\hline Histórico & $\begin{array}{c}\text { CEPA, Tironi, } \\
\text { Sorj. }\end{array}$ & $\begin{array}{c}\text { Conflicto social, } \\
\text { desintegración, } \\
\text { desarticulación. }\end{array}$ & $\begin{array}{c}\text { Vínculos horizontales } \\
\text { y verticales, Sentido } \\
\text { de pertenencia. }\end{array}$ \\
\hline Sociológico & $\begin{array}{c}\text { Dorkheim, } \\
\text { To y Chan, }\end{array}$ & $\begin{array}{c}\text { Disolución, inestabilidad, } \\
\text { desintegración social. }\end{array}$ & $\begin{array}{c}\text { Lazos, confianza, } \\
\text { valores, sentido de } \\
\text { pertenencia. }\end{array}$ \\
\hline
\end{tabular}

Fuente: elaboración propia, con base en Vera (2014) y Nari (2015).

Sin embargo, en general la literatura de la cohesión social se refiere a la búsqueda de comunidad, dentro de una situación en la que la estructura social se encuentra fracturada y se están debilitando las formas tradicionales de los vínculos sociales, para dar paso a un proceso de predominancia de lo individual sobre lo colectivo (CEPAL, 2007). 
Al ser este el atributo que mantiene unida a una sociedad, alcanzarla significa lograr otras metas sociales, como el desarrollo económico, el desarrollo humano y el desarrollo sostenible, como afirma Medina (2016), que la cohesión social es el elemento indispensable para el funcionamiento de una sociedad, el pegamento que la mantiene unida y que solo en su búsqueda se logrará rescatar a la sociedad.

De esta manera, la organización Cohesión Comunitaria e Innovación Social (2010), indica que alcanzar la cohesión tiene un efecto positivo en diferentes aspectos como aumento en la satisfacción de vida, el desempeño educativo, la inversión privada; y disminuye problemas sociales como la violencia, la deserción escolar, enfermedades mentales, suicidios, estrés, depresión, entre otros.

Si un país como una comunidad cuenta con una baja cohesión social, se manifestarán problemas sistemáticos como la violencia, la injusticia, el delito y la impunidad, que se resume en una inestabilidad estructural con una ausencia de desarrollo, de lo contrario, si cuenta con una elevada cohesión social, se facilita el crecimiento económico y con ello el desarrollo, la competitividad, potencial de innovación, gobernabilidad y disminución de las brechas socioeconómicas (Guerrero, Franco y Bass, 2016).

Indiscutiblemente, la cohesión social es un atributo de la naturaleza humana, pues al erigirse el hombre como un animal político, no es posible concebir una sociedad sin alguna forma de cohesión, pues simplemente esta no existiría. De aquí la importancia, pues contra aquellos factores que buscan romper el tejido de la sociedad, es la cohesión social la que busca reestablecerlo.

\section{ESTRATEGIAS METODOLÓGICAS}

De acuerdo a Hernández (2014), el tipo de estudio es correlacional, de corte longitudinal (periodo de tiempo de dos años), cuasi-experimental y pretendió a través de la participación ciudadana influir en la cohesión social de la comunidad. Encontró en el enfoque cualitativo la metodología indicada para poder alcanzar el objetivo de la investigación, ya que, al buscar la realización de una intervención en una comunidad, esta se instituyó como un proyecto meramente social, donde la interacción con las personas fue su base fundamental, ya que tuvo como esencia principal "ir hacia la gente" (como indican Taylor y Bogdan, 1987; p. 15.), convirtiéndose en un estudio humanista.

Por lo tanto, la intervención se llevó a cabo a partir de la metodología "investigación acción", la cual de acuerdo a Vidal y Rivera (2007), es una forma de indagación 
introspectiva y colectiva, emprendida por participantes en situaciones sociales con objeto de mejorar la racionalidad y la justicia de sus prácticas sociales, así como su comprensión de esas prácticas y de las situaciones en que estas tienen lugar.

Para López de Méndez (2012), la investigación acción, tiene como propósito el promover el cambio y hacer transformaciones en situaciones reales, contribuyendo al bien social. Sandín (2003) señala que ésta pretende propiciar el cambio social, transformar la realidad y que las personas tomen conciencia de su papel en ese proceso de transformación. Se utiliza cuando se desea mejorar el entendimiento o conocimiento de un fenómeno, a la vez que se busca influenciar a otros para la solución de un problema práctico.

Dentro de la investigación acción se adaptó la clasificación de la investigación acción participativa, basada en la propuesta de Bertomeu y Sabariego (2018), los cuales señalaron que este es un enfoque mediante el que se pretende la plena participación de la comunidad en el análisis de su propia realidad, con el objeto de promover la transformación social para beneficio de los participantes de la investigación a nivel de la comunidad.

Así mismo, en base a la propuesta de intervención comunitaria de Mori (2008), se determinaron las cuatro siguientes etapas de la intervención:

\section{Diagnóstico.}

Fue de donde partió la intervención, en la que se hizo un examen preliminar de la comunidad, basándose en una revisión crítica del estado de situación existente. Tuvo como objetivo identificar, analizar y describir las condiciones en la que se desenvuelve la comunidad y así tener las bases necesarias para el diseño de una intervención en la organización.

\section{Diseño del Plan.}

De acuerdo a las necesidades detectadas, se pasó a determinar las posibles soluciones, que incluyeran la participación de los propios habitantes. En este punto se definieron los objetivos, las metas, los recursos, el mecanismo de seguimiento y en general el desglose de actividades a realizar con el objeto de transformar la realidad en la que vive la comunidad.

\section{Implementación del Plan de Acción.}

Esta es la fase operativa, en la que se llevó a la práctica lo planteado en la fase anterior, fue la puesta en marcha de las estrategias decididas por el equipo de la intervención. 
Esta fue la etapa más prolongada en tiempo, pues es la parte principal, donde tiene su desarrollo natural la investigación acción y cumpliendo con el objetivo de la investigación de llevar a cabo la intervención.

\section{Evaluación y Retroalimentación.}

Una vez agotado el curso de acción, se pasó a la evaluación final, donde se pasó a determinar el resultado de la intervención, respondiendo a la pregunta de investigación y poniendo a prueba la hipótesis del estudio, para concluir con el reporte final.

Cada etapa de la intervención se implementó con el apoyo de las siguientes técnicas de indagación y recopilación de datos:

\section{Observación participante.}

Consistente en hacerse parte de la comunidad, investigando sus hechos y acontecimientos, para interpretar el comportamiento social (Martínez, 2004). "Implica adentrarse profundamente en situaciones sociales y mantener un papel activo, así como una reflexión permanente. Estar atento a los detalles, sucesos, eventos e interacciones" (Hernández, 2014, p. 399). Se centra en la interacción entre el investigador y el sujeto, a medida que se recopila la información de los hechos que van sucediendo (Taylor y Bogdan, 1987).

\section{Entrevista a profundidad.}

Es una interacción o dialogo bidireccional, que Hernández (2014), la define como una reunión para conversar e intercambiar información entre una persona y otra, en la que mediante preguntas y respuestas se logra una construcción conjunta de significados respecto a un tema. El propósito es obtener "descripciones del mundo vivido", con el fin de lograr "interpretaciones fidedignas" del significado que tienen los fenómenos. En esta se pueden hacer preguntas sobre experiencias, opiniones, valores, creencias, emociones, sentimientos, hechos, historias de vida, percepciones, atribuciones, etcétera.

\section{Grupo de enfoque.}

Es la técnica cualitativa por excelencia en la que se busca el diálogo y la participación de las personas objeto de estudio, creando un ambiente interactivo y dinámico (Báez y Pérez, 2012), propio de la naturaleza del presente estudio. Es "focal" porque concentra su atención e interés en un tema específico de estudio e investigación, y es de "discusión" porque realiza su principal trabajo de búsqueda por medio de la interacción discursiva y la contrastación de las opiniones de sus miembros. Es un método de investigación 
colectivista, más que individualista, y se centra en la pluralidad y en la variedad de las actitudes, las experiencias y las creencias de los participantes (Martínez, 2004).

Cabe destacar que la implementación del Plan de Acción, se llevó a cabo en tres ciclos, los cuales a su vez fungieron como los bucles de la investigación acción propuestos por Lewin (1946) y Carr y Kemmis (1988), debido a que atendieron el crecimiento gradual del proceso de la participación ciudadana y su influencia en la cohesión social de la colonia, por lo que se propusieron en tres tiempos de evaluación y retroalimentación del proyecto. Dichos ciclos atendieron el siguiente orden y respectivo propósito:

1. "Acción de la comunidad". - Sensibilizar a la población para su "involucramiento" en la solución de la problemática de su comunidad.

2. “Acción de agentes externos". - Motivar a la población de su "colaboración" formal con la sociedad civil organizada.

3. "Empoderamiento comunitario". - Consolidar una comunidad organizada, autosuficiente y "empoderada".

La propuesta conceptual de la participación ciudadana, seleccionada y adaptada para la presente intervención fue la de IAP2 (2018), la cual indica que esta es cualquier proceso que involucra al público en la resolución de problemas y en la toma de decisiones; y desglosa sus diferentes dimensiones en:

- Informar.- En donde el ciudadano se informa para entender los problemas de su entorno.

- Consultar.- En donde el ciudadano obtiene retroalimentación pública para generar sus propias opiniones y las expresa.

- Involucrar.- En donde el ciudadano trabaja directamente con el público para asegurar que sus preocupaciones y aspiraciones, se comprendan y se consideren.

- Colaborar.- En donde el ciudadano comprometidamente se asocia con la comunidad para influir en el desarrollo de su entorno.

- Empoderar.- En donde el ciudadano de manera formal toma decisiones junto a la comunidad.

Así mismo la propuesta conceptual de la cohesión social, que se adoptó y adecuó para la presente intervención, fue la elaborada por Mora (2015, p.117), el cual señala que esta es "la existencia de una estructura de vínculos sociales y la disposición de los individuos a mantener y renovar dichos lazos sociales, la identificación de los individuos con la 
colectividad y la presencia de valores compartidos". El autor propone las siguientes dimensiones de la cohesión social:

- Lazos sociales. - Se entiende la existencia y reproducción de relaciones sociales formadas a partir de la interacción cotidiana recurrente entre individuos miembros de una colectividad.

- Confianza. - Se entiende la expectativa de comportamiento futuro de las personas con las que un individuo mantiene relaciones o podría llegar a establecer vínculos.

- Valores compartidos. - Conjunto de principios y motivaciones generales que orientan los juicios, actitudes y comportamientos de los individuos.

- Sentido de pertenencia. - Se entiende la existencia del sentimiento de formar parte de una colectividad, a sentirse miembro de una sociedad, identificarse con sus rasgos fundamentales y tener un futuro compartido.

\section{RESULTADOS Y DISCUSIÓN}

Se hizo un análisis comparativo de los resultados de la etapa de diagnóstico con los resultados de la evaluación de la intervención, en donde se reflejan los niveles alcanzados de las variables en estos dos momentos de medición, el primero antes de la intervención y el segundo después de esta, en base a los postulados conceptuales mencionadas de la participación ciudadana de IAP2 (2018) y de la cohesión de social de Mora (2015).

Para el análisis de los datos se realizó una sistematización analítica en base a Rådiker y Kuckartz (2020), por medio del software de procesamiento de datos cualitativos MAXQDA®, la cual consistió en una categorización, análisis de frecuencias y concurrencias e interpretación de la información en dos tiempos como se mencionó, antes y después de la intervención. Así mismo, se propuso una escala de medición del nivel de presencia de cada variable en los dos momentos de análisis mencionados y en base a la frecuencia obtenida por cada una de sus respectivas dimensiones, se determinaron medirlos de acuerdo a la escala:

$0=$ nulo,

1 = muy bajo,

2 = bajo,

$3=$ medio,

$4=$ alto $\mathrm{y}$

5 = muy alto; quedando como indica la tabla 2. 
Tabla 2. Nivel de presencia de variables, en etapas de diagnóstico y evaluación de la intervención.

\begin{tabular}{|l|l|c|c|}
\hline \multirow{2}{*}{ Variable } & \multicolumn{1}{|c|}{ Dimensión } & \multicolumn{2}{c|}{ Nivel } \\
\cline { 3 - 4 } & & Antes & Después \\
\hline \multirow{4}{*}{$\begin{array}{l}\text { Participación } \\
\text { Ciudadana }\end{array}$} & Informar & 2 & 3 \\
\cline { 2 - 4 } & Consultar & 1 & 3 \\
\cline { 2 - 4 } & Involucrar & 0 & 4 \\
\cline { 2 - 4 } & Colaborar & 0 & 5 \\
\cline { 2 - 4 } & Empoderar & 2 & 3 \\
\hline \multirow{4}{*}{ Cohesión Social } & Lazos sociales & 1 & 4 \\
\cline { 2 - 4 } & Confianza & 0 & 1 \\
\cline { 2 - 4 } & Valores compartidos & 0 & 2 \\
\cline { 2 - 4 } & Sentido de pertenencia & & \\
\hline
\end{tabular}

Fuente: elaboración propia.

Se puede observar que de manera general hubo un aumento en todas las dimensiones de las dos variables al final de la intervención. Realizando una comparativa gráfica de la variable participación ciudadana en los dos momentos de la intervención (figura 1), se puede observar que hubo un aumento sustancial en todas las dimensiones, antes de la intervención (etapa de diagnóstico), la dimensión con más frecuencia era la de "informar", en menor medida "consular"; y muy bajo, al punto nulo, "involucrar", "colaborar" y "empoderar"; después de la intervención (etapa de evaluación), la dimensión más frecuente fue "colaborar", seguido por "empoderar" e "involucrar", y en abajo "consultar" e "informar".

Figura 1. Gráfica de radar de participación ciudadana.

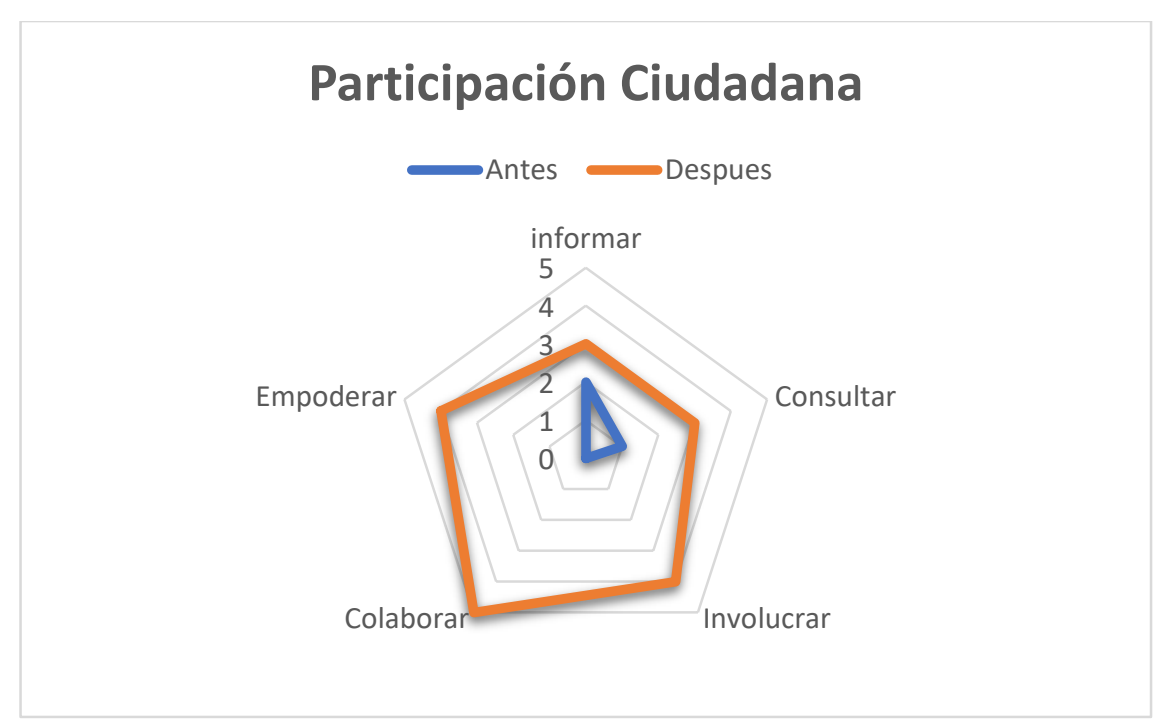

Fuente: elaboración propia. 
De la misma manera, en la figura 2, podemos ver de manera notoria que la cohesión social de igual forma, tuvo un aumento radical en la comunidad, antes de la intervención (etapa de diagnóstico), la dimensión con más presencia era "lazos sociales", muy bajo la “confianza" y nulos los "valores compartidos" y el "sentido de pertenencia"; después de la intervención, (etapa de evaluación), podemos ver que la dimensión más presente, fue la de "confianza", seguido de cerca por "lazos sociales", y más abajo, el "sentido de pertenencia" y "valores compartidos".

Figura 2. Gráfica de radar de cohesión social.

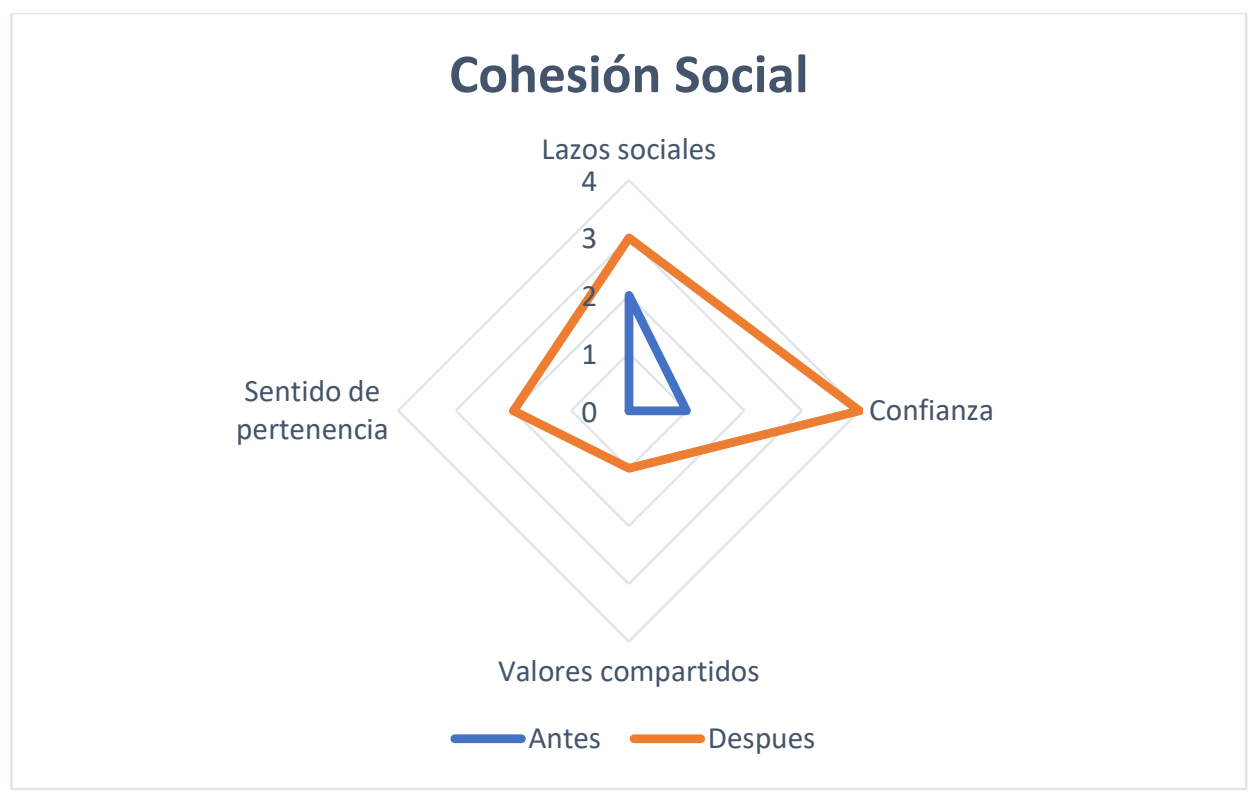

Fuente: elaboración propia.

En resumen, es tangible que la intervención tuvo un efecto de cambio en la comunidad motivado por el aumento de la presencia de la participación ciudadana y de la cohesión social. Como se puede observar en la tabla (3), las condiciones no son las mismas, al inicio se lograba notar una colonia prácticamente sin interés ni relaciones entre sus habitantes; ahora se puede notar como una comunidad, en donde domina el entendimiento y el acuerdo. 
Tabla 3. Resumen comparativo de la intervención en la comunidad.

\begin{tabular}{|c|c|c|}
\hline & & \\
\hline $\begin{array}{l}\text { Las condiciones en las que se } \\
\text { encontraba la colonia eran de } \\
\text { desintegración, división y } \\
\text { pesimismo, en donde } \\
\text { respecto a la participación } \\
\text { ciudadana, se limitaban a un } \\
\text { nivel de "informarse", pues } \\
\text { les era indiferente } \\
\text { relacionarse con los demás y } \\
\text { se enteraban de lo sucedido a } \\
\text { su alrededor por propia } \\
\text { seguridad. En la cohesión } \\
\text { social, había un ambiente } \\
\text { generalizado de desconfianza } \\
\text { entre vecinos, motivo por el } \\
\text { cual, los lazos sociales se } \\
\text { limitaban sólo a los que se } \\
\text { pudieran dar dento del hogar. }\end{array}$ & $\begin{array}{l}\text { La intervención se llevó a } \\
\text { cabo a partir de tres etapas } \\
\text { en las que se realizaron } \\
\text { principalmente las } \\
\text { siguientes acciones: } \\
\text { 1. Acción de la } \\
\text { comunidad: visita a } \\
\text { cada casa, recreación, } \\
\text { reuniones } \\
\text { informativas, apoyos y } \\
\text { rescate de espacios } \\
\text { públicos. } \\
\text { 2. Acción de agentes } \\
\text { externos: brigadas de } \\
\text { atención integral y } \\
\text { gestión } \\
\text { autoridades. } \\
\text { 3. Empoderamiento: } \\
\text { conformación } \\
\text { comité vecinal y plan } \\
\text { de vigilancia. }\end{array}$ & $\begin{array}{l}\text { Las condiciones que } \\
\text { presenta la colonia en este } \\
\text { momento son de } \\
\text { integración, unión y } \\
\text { optimismo, en donde } \\
\text { respecto a la participación } \\
\text { ciudadana se encuentra en } \\
\text { un nivel de "colaboración", } \\
\text { pues los miembros } \\
\text { muestran un compromiso } \\
\text { formal como comunidad y } \\
\text { la intención de seguir } \\
\text { trabajando por el desarrollo } \\
\text { de su colonia. En la } \\
\text { cohesión social, } \\
\text { presenta una "confianza" } \\
\text { generalizada entre vecinos, } \\
\text { donde se da el trabajo } \\
\text { colaborativo, relaciones de } \\
\text { amistad y momentos de } \\
\text { convivencia. }\end{array}$ \\
\hline
\end{tabular}

Fuente: elaboración propia.

Esta transformación fue posible por las acciones realizadas en la intervención comunitaria, estas buscaron atender un proceso gradual en la búsqueda de participación ciudadana y cohesión social, por medio de tres etapas, en las que se primeramente de sensibilizó a la población sobre la importancia de transformar su entorno y la importancia de su "involucramiento" para ello, para lograrlo fue fundamental el rapport por medio de estrategias que lograran hacer una conexión con la población, con acciones como las que se muestran en la tabla; continuando a la siguiente etapa, se logró la "colaboración" de la gente en la actividad de la sociedad civil organizada, para que se instruyeran en el modo de trabajar de manera organizada y colectiva, para lograr esto, se invitó a la Universidad 
Juárez del Estado de Durango para que trabajara en la colonia el programa de brigadas universitarias en el que la participación de la gente de la colonia fue fundamental; finalmente, en la última etapa, se crearon las condiciones para que la población alcanzara su "empoderamiento", para alcanzarlo se conformaron mecanismos formales de participación ciudadana y trabajo comunitario, como la puesta en marcha del consejo vecinal para la toma de decisiones y el plan de vigilancia para combatir la inseguridad. Es importante destacar que las acciones realizadas, fueron planeadas y llevadas a cabo junto con la gente, haciéndose parte de la intervención de su propia comunidad y siendo para la investigación mas que un objeto de estudio, un sujeto de estudio.

Con esto aumentó el contacto entre las personas por lo que mejoraron sus relaciones, se manifiesta en ellos un sentimiento de orgullo de formar parte de la colonia, un sentido de solidaridad, cooperación y altruismo, aceptación por las opiniones de los demás, un reconocimiento de los demás a su labor y valor como individuo, así como un deseo explícito de seguir trabajando unidos como comunidad.

\section{CONCLUSIÓN}

De acuerdo al objetivo del estudio se confirma que a partir de la intervención hubo un cambio en la colonia, un aumento en su participación como individuos y como comunidad; y un aumento en los factores que la cohesionan. Obtuvo un cambio visible en sus condiciones sociales, ya que anteriormente no existían ni las relaciones mínimas entre personas, al grado de no dirigirse la palabra o un signo de cortesía entre ellos.

La colonia ha cambiado de un ambiente negativo por uno lleno de participación, trabajo, generosidad y orgullo de ser parte ahora de una comunidad. Si bien no se alcanzó el nivel más alto de la participación ciudadana de acuerdo a la escalera de la participación, se muestra que estuvo a un ligero paso para lograrlo, debido a que, si bien se cuenta con el Consejo Vecinal sesionando y una aportación activa de las personas en las decisiones de la colonia, aun se tiene un cierto sentido de apego y dependencia al equipo de la investigación, para seguir trabajando en comunidad como se ha logrado.

Así mismo, aunque no se alcanzó un nivel máximo de cohesión social, se puede ver con satisfacción que esta aumentó considerablemente comparado con la situación inicial, la colonia ya no es la misma, cambió en su comportamiento, forma de comunicarse y de ver el futuro. La gente no encontraba una razón de esforzarse por mejorar su entorno, se vivía en una forma egoísta o individual de ver la vida y ahora la gente vive en una común- 
unidad, donde la opinión de cada uno cuenta, se sienten parte de un equipo en el que comparten un sentido de futuro compartido, donde acusan que antes no se conocían entre las personas y ahora se han generado círculos de amistad, en esto jugó un papel importante la vinculación que se logró en la convivencia y recreación, pues permitió que las personas aprendieran a conocer al otro y dejaran al lado los prejuicios.

De esta manera, de acuerdo a la pregunta de investigación ¿de qué manera influye la participación ciudadana en la cohesión social de la comunidad? Podemos afirmar que la participación ciudadana tiene influencia directa en la cohesión social de un grupo u organización, y esta se da en la medida en que las personas se involucran en un proyecto para beneficio en común, aumentando los atributos que los pueden unir e identificarlos como parte de un conjunto social.

En cuanto a las hipótesis de investigación, que indica: "el aumento de la participación ciudadana de la población, en los asuntos de su propio entorno, genera un incremento en la cohesión social de la Colonia Legisladores de la ciudad de Durango, México", se establece que se aprueba. Aunado a esto, se puede agregar que tres factores fueron determinantes para que se pudiera lograr un cambio en la colonia: el "liderazgo" que tuvo el equipo de investigación sobre la gente, el "espacio público" que fungió como el punto de encuentro entre las personas para la convivencia y el acuerdo; y la "disminución de la inseguridad", que es considerado por los colonos, como uno de los mayores logros de la intervención.

De esta manera, se concluye que en la medida en que los ciudadanos se hagan partícipes de manera comunitaria del mejoramiento de las condiciones de su entorno, aumentarán sus lazos, su confianza, sus valores y su sentido de pertenencia. Concibiendo la cohesión social como un constructo que se da desde la sociedad y no desde la individualidad, sin depender necesariamente de la iniciativa y la intervención del gobierno o de actores externos.

Finalmente, se puede confirmar que el cambio en la comunidad se dio, ya no es la misma de antes, se ha transformado, la gente ha cambiado su actitud y ahora se encuentra en disposición de crecer comunitariamente. La comunidad de la Colonia Legisladores ha encontrado un sentido en este proyecto, una transformación en su forma de pensar y vivir, por lo que esta intervención, ha puesto de manifiesto que con la participación ciudadana de una comunidad puede aumentar su cohesión social. 


\section{LISTA DE REFERENCIA}

Alberich, T., \& Espadas, M. (2014). Democracia, participación ciudadana y funciones del trabajo social. Revista de Investigaciones en Intervención Social, 3-30.

Aguilar, L. (2007). El aporte de la Política Pública y de la Nueva Gestión Pública a la Gobernanza. CLAD, 1-15.

Baez y Pérez, J. (2012). Investigación cualitativa. Madrid: Alfaomega.

Borja, J. (2000). El espacio público, ciudad y ciudadanía. Barcelona: Borjaespacio.

Carr, W., \& Kemmis, S. (1983). Teoría crítica de la enseñanza: La investigación acción en la formación del profesorado. Barcelona: Ediciones Martinez Roca.

Cattani, A. (2011). Cohesión social y políticas públicas sin molestar a los sectores dominantes. Buenios Aires: CLACSO.

CEPAL. (2007). Cohesión Social: Inclusión y sentido de pertenencia en AméricaLatina y el Caribe. Santiago de Chile: Naciones Unidas.

Cohesión Comunitaria e Innovación Social. (2010). Del tejido social a la cohesion comunitaria: una aproximación inicial para México. Ciudad de México: Este País.

Cunill, N. (1991). Participación ciudadana. Venezuela: Editorial CLAD.

Folgueiras Bertomeu, P., \& Sabariego Puig, M. (2018). Investigación-acción participativa. El diseño de un diagnóstico participativo. REIRE, 16-25.

Gomez Romero, J. (2016). El liderazgo de aprendizaje y su incidencia en la innovación y competitividad de las mipymes de la ciudad de Durango, México. Investigación Administrativa, 52-67.

Guerrero, J., Franco, L., \& Bass, S. (2016). El sistema de cohesión social y sus efectos en la tasa de delicuencia. Revista Ciencia Ergo Sum, Pp. 5-16.

Guerrero, M. (2015). Cohesión social, razón y gobernanza . Cuernavaca, Morelos: Fontamara.

Hall, R. H. (1996). Organizaciones: Estructura y procesos. Madrid, España: PrenticeHall, Inc.

Hernández, S. R. (2014). Metodología de la Investigación. México D.F.: Mc Graw Hill. IAP2. (2018). IAP2 Spectrum of Public Participation. Londres: International Association for Public Participation. 
Jiménez, W. (2013). La violencia y cohesión social: una aproximación a la construcción de un índice de cohesión social. Revista Diereuti da Cidade, Pp. 483-512.

Lastra, G. (11 de Mayo de 2017). Alcalde de Durango asegura que han variado los índices de inseguridad en la ciudad. Multimedios, pág. 2.

Lechner, N. (1991). El ciudadano y la noción de lo público. Revista de hechos e ideas, 43,44 .

Lewin, K. (1946). Action research and minority problems. Journal of Social Issues, 2, $34-46$.

López de Méndez, A. (2012). Investigacion Acción. Una alternativa para fortalecer la investigacion del proceso de enseñanza y aprendizaje (págs. 1-30). Puerto Rico: Universidad de Puerto Rico.

Martinez Miguélez, M. (2004). Ciencia y arte de la metodología cualitativa. México, D.F.: Trillas.

Medina, E. (2016). Cohesión social y democracia: una nueva formulación para la construcción del desarrollo en un mundo globalizado. Sao Paulo: Universidad de Sao Paulo.

Mora, M. (2015). Cohesión social: balance conceptual y propuesta metodológica. Cdmx: CONEVAL.

Mori, M. D. (2008). Una propuesta metodológica para la intervención comunitaria . LIBERABIT, 14: 81-90.

Nari, P. (2015). Ciudades des-tramadas: Politicas locales de Cohesión Social Urbana. Un estudio comparado de politicas publicas locales en territorios excluidos y aportes de diseño. Valencia: Universidad Politécnica de Valencia.

Oliván Cortés , R. (2014). La Cuarta Revolucion Industrial, un relado desde el materialismo cultural. Revista de Estudios Urbanos y Ciencias Sociales, 101-111.

Olvera, A. (2019). Ciudadanía y democracia. Ciudad de México: Instututo Nacional Electoral.

Paz y Miño, S. (2017). La deshumanización de la sociedad. Razón y Palabra, 688-697.

Rådiker, S., \& Kuckartz, U. (2020). Análsis de Datos Cualitativos con MAXQDA. Berlín: MAXQDA Press.

Sandín Esteban, M. P. (2003). Investigación Cualitativa en Educación: Fundamentos y Tradiciones. Madrid: Mac Graw and Hill Interamericana. 
Taylor, S., \& Bodgan, R. (1987). Introducción a los métodos cualitativos de investigación. Buenos Aires: Ediciones Paidos Ibérica S. A.

Vargas, J. G. (2007). Crisis de la Gobernabilidad del Estado-Nación. Los problemas del mundo actual: soluciones y alternativas desde la Geografía y las Ciencias Sociales, 1-14.

Vera Alpuche, J. (2014). Nucleo y dimensiones de la cohesión social, enfoques contemporaneos. 2-22.

Vidal Ledo, M., \& Rivera Michelena, N. (2007). Investigación acción. La Habana: Escuela Nacional de Salud Pública.

Zimmerman, J. (1992). Democracia participativa: el resurgimiento del populismo. México: Limusa . 